\title{
ESTIMATIVAS DE CHUVAS INTENSAS PARA O ESTADO DE GOIÁS
}

\section{LUIZ F. C. DE OLIVEIRA ${ }^{1}$, JORGE C. DOS A. ANTONINI ${ }^{2}$, NORI P. GRIEBELER ${ }^{3}$}

RESUMO: A ausência de estações pluviográficas e de séries históricas longas tem levado os Engenheiros à utilização de metodologias que permitam expressar a relação intensidade-duraçãofreqüência de precipitações críticas. Com o objetivo de estimar as alturas de chuvas intensas, associadas a uma duração e freqüência, desenvolveu-se uma rotina computacional para ajustar os parâmetros do modelo de Bell para alguns municípios do Estado de Goiás. Para tal, empregaram-se séries históricas de precipitações diárias e de relações intensidade-duração-freqüência disponíveis para alguns municípios. Determinou-se, também, a relação entre a precipitação de 60 minutos e 1 dia de duração, para um período de retorno de dois anos. As informações geradas neste trabalho foram regionalizadas, permitindo a geração de mapas temáticos, visando a estimar a relação altura precipitada-duração-freqüência para as localidades desprovidas de registros. Para os municípios estudados, o modelo de Bell ajustou-se perfeitamente, apresentando alternativa interessante na obtenção das alturas de chuvas intensas a partir de séries curtas. A reconstrução do modelo de Bell a partir da regionalização dos parâmetros ajustados permitiu a ampliação das equações que expressam a relação entre a precipitação máxima para diferentes durações e o período de retorno com baixos valores no erro-padrão da estimativa.

PALAVRAS-CHAVE: modelo de Bell, drenagem, escoamento superficial.

\section{INTENSE RAINFALL ESTIMATES FOR THE GOIÁS STATE, BRAZIL}

\begin{abstract}
The absence of pluviograph stations and of long historical series has induced engineers to use methodologies that allow them to express the intensity-duration-frequency relation of critical rainfalls. With the purpose of estimating the height of intense rain associated to a given duration and frequency, it was developed a computational routine to adjust the parameters of the Bell's model to some municipal districts of the Goiás State. For such work it was employed historical series of daily precipitation and intensity-duration-frequency relations available to some municipal districts. It was also determined the relation between the precipitation of sixty minutes and one day duration for two years return period. The information obtained in this work was regionalized, allowing the generation of thematic aiming to estimate the rainfall height-durationfrequency relation for the places without registrations. The Bell's model perfectly adjusted to the municipal districts that were studied, becoming an interesting alternative concerning to acquiring the height of intense rain from short series. The reconstruction of Bell's model based on the regionalization of the adjusted parameters allowed the expansion of the equations that express the relation between the maximum precipitation for different durations and the return period with low values in the standard error of the estimate.
\end{abstract}

KEYWORDS: Bell's model, drainage, runoff.

\footnotetext{
${ }^{1}$ Professor Adjunto, Universidade Federal de Lavras, Departamento de Engenharia, Caixa Postal 3037, Lavras - MG, 1fco@pq.cnpq.ufg.br. Bolsista em produtividade do CNPq.

2 Pesquisador da EMBRAPA-Transferência de Tecnologia, Doutorando Solo e Água-UFG, jorge.antonini@embrapa.br.

${ }^{3}$ Professor Adjunto, Escola de Agronomia e Engenharia de Alimentos, Universidade Federal de Goiás, nori@agro.ufg.br.

Recebido pelo Conselho Editorial em: 14-12-2006
}

Aprovado pelo Conselho Editorial em: 14-11-2007 


\section{INTRODUÇÃO}

Segundo COSTA et al. (2001), para a utilização prática e adequada dos dados de chuva, na elaboração de projetos de drenagem, barragens e obras de proteção contra cheias e erosão hídrica, faz-se necessário conhecer a relação intensidade-duração-freqüência das chuvas intensas. Vários modelos matemáticos foram propostos para expressar essa relação, com parâmetros ajustados pelo método dos mínimos quadrados a partir de informações extraídas de pluviogramas, dentre os quais estão o de BELL (1969), PFAFSTETTER (1957), GENOVEZ \& ZUFFO (2000) e COSTA et al. (2001).

Segundo SILVA et al. (1999a, 1999b), MARTINEZ JÚNIOR (1999) e COSTA \& BRITO (1999), a determinação da relação intensidade-duração-freqüência, expressa pela eq.(1), apresenta grandes dificuldades em função da escassez de registros pluviográficos, da baixa densidade da rede de pluviógrafos e do curto período de observações disponível. Além disso, a metodologia para sua obtenção exige exaustivo trabalho de tabulação, análise e interpretação de grande quantidade de pluviogramas (OLIVEIRA et al., 2005). A intensidade de uma chuva, associada a sua duração e freqüência de ocorrência, pode ser expressa como:

$$
\mathrm{i}_{\text {máx }}=\frac{\mathrm{aTR}^{\mathrm{b}}}{(\mathrm{t}+\mathrm{c})^{\mathrm{d}}}
$$

em que,

$\mathrm{i}_{\text {máx }}$ - intensidade máxima média, $\mathrm{mm} \mathrm{min}^{-1}$;

TR - período de retorno, anos;

$\mathrm{t}$ - tempo de duração da chuva, minutos, e

a, b, c e d - parâmetros de ajuste obtidos pelo método dos mínimos quadrados.

O trabalho clássico de estudos de chuvas intensas no Brasil foi publicado por PFAFSTETTER (1957). Na literatura, os mais recentes são os de FENDRICH (1998), para o Estado do Paraná, PINTO et al. (1999), para o Estado de Minas Gerais, COSTA \& BRITO (1999), para o Estado de Goiás e duas cidades de Tocantins, SILVA et al. (1999a, 1999b, 2002, 2003), para os Estados de São Paulo, Rio de Janeiro e Espírito Santo, Bahia e Tocantins, respectivamente, e OLIVEIRA et al. (2005), para o Estado de Goiás e Distrito Federal.

Algumas metodologias foram desenvolvidas no Brasil para a obtenção de chuvas de menor duração a partir de registros pluviométricos diários, devido à existência no território nacional de vasta rede pluviométrica. Essas metodologias empregam coeficientes para transformar chuva de 24 horas, em chuvas de menor duração. Dentre elas, estão a das isozonas e a da desagregação da chuva de 24 horas, citadas por OLIVEIRA et al. (2005). COSTA \& RODRIGUES (1999) compararam os resultados gerados com o método das isozonas com os obtidos por meio da relação intensidadeduração-freqüência, encontrando desvios entre 7,5\% e 54,0\%. BARBOSA et al. (2000) empregaram a metodologia da desagregação da chuva de 24 horas para algumas localidades do Estado de Goiás, a qual se mostrou adequada, com valores de desvios menores que $14,4 \%$, comparados com as relações intensidade-duração-freqüência geradas por COSTA \& BRITO (1999). Os autores recomendaram o uso da metodologia em localidades em que não há disponibilidade de registros pluviográficos. Por outro lado, OLIVEIRA et al. (2005) ajustaram para algumas localidades do Estado de Goiás e do Distrito Federal a relação intensidade-duração-freqüência, empregando o método de desagregação de chuvas de 24 horas. Os resultados obtidos pelas relações ajustadas apresentaram desvios relativos médios que variaram de $-1,6 \%$ a $43,9 \%$.

Uma alternativa para a obtenção da altura pluviométrica de chuva intensa associada a uma determinada duração e freqüência de ocorrência consiste na utilização do método de BELL (1969). Segundo BERTONI \& TUCCI (1993) e RIGHETTO (1998), o método de Bell associa a altura pluviométrica de uma chuva intensa para um determinado tempo de duração e período de retorno à chuva intensa, padrão de 60 minutos de duração, e dois anos de período de retorno eq.(2). 


$$
\mathrm{h}_{(\mathrm{t} ; \mathrm{TR})}=\left(\alpha \ln \mathrm{TR}+\beta_{1}\right)\left(\beta_{2} \mathrm{t}^{\gamma}-\beta_{3}\right) \mathrm{h}_{(60 ; 2)}
$$

em que,

$\mathrm{h}_{(\mathrm{t} ; \mathrm{TR})}$ - altura pluviométrica $(\mathrm{mm})$ de chuva intensa de duração t e período de retorno TR;

$\mathrm{h}_{(60 ; 2)}$ - altura pluviométrica $(\mathrm{mm})$ de chuva intensa, padrão de 60 minutos de duração, e dois anos, e

$\alpha, \gamma, \beta_{1}, \beta_{2}$ e $\beta_{3}$ - parâmetros regionais ajustados pelo método dos mínimos quadrados.

O método só se aplica se for possível estimar $\mathrm{h}_{(60 ; 2)}$, o que pode ser feito com poucos anos de dados pluviográficos. Inexistindo observações pluviográficas, porém dispondo-se de dados de precipitações máximas anuais com duração diária, pode-se estimar $\mathrm{h}_{(60 ; 2)}$ recorrendo à relação empírica [eq.(3)]:

$$
\mathrm{h}_{(60 ; 2)}=\mathrm{K} \mathrm{h}_{(\mathrm{dia} ; 2)}
$$

em que,

K- relação regional entre a precipitação pluvial de 60 minutos e um dia de duração para um período de retorno de dois anos.

Empregando-se as relações intensidade-duração-freqüência existentes na literatura e as séries históricas disponíveis de chuvas máximas anuais, foi desenvolvida uma rotina computacional para ajustar os parâmetros do modelo de Bell para algumas localidades do Estado de Goiás. Para permitir a obtenção da altura pluviométrica associada ao tempo de duração e freqüência de ocorrência, para localidades sem registros de chuva, os parâmetros ajustados foram regionalizados, permitindo a reconstrução do modelo de Bell para qualquer localidade inserida no Estado de Goiás. Os resultados gerados neste trabalho possibilitam aos Engenheiros a obtenção das informações básicas para a elaboração de projetos de obras de drenagem no Estado de Goiás.

\section{MATERIAL E MÉTODOS}

No ajuste dos parâmetros do modelo de Bell, foram selecionadas as estações do Estado de Goiás dotadas de registros de pluviógrafos, para as quais foram ajustadas as relações intensidadeduração-freqüência. Os parâmetros da eq.(1), apresentados na Tabela 1, foram ajustados por COSTA et al. (2001) e OLIVEIRA et al. (2005), empregando o método dos mínimos quadrados e as informações extraídas de pluviogramas.

Seguindo a recomendação de BERTONI \& TUCCI (1993), de que o método de Bell só se aplica em chuvas com durações menores que 120 minutos e períodos de retorno inferiores a 20 anos, determinaram-se as intensidades médias e as alturas pluviométricas máximas para os tempos de duração de 5; 10; 30; 60; 90 e 120 minutos, e períodos de retorno de 2; 5; 10 e 20 anos.

O modelo de Bell, descrito pela eq.(2), é uma função não-linear que não possibilita sua linearização por transformação logarítmica. Nesse caso, procurou-se associar ao método dos mínimos quadrados, técnicas numéricas para a solução de modelos não-lineares. Para tal, o modelo de Bell foi descrito pela função $\mathrm{f}\left(\alpha, \gamma, \beta_{1}, \beta_{2}, \beta_{3}\right)$, que estima o valor da variável dependente $\mathrm{h}_{\mathrm{t} \text {;TR })}$ em relação aos assumidos para as variáveis independentes $\mathrm{TR}, \mathrm{t}$ e $\mathrm{h}_{(60 ; 2)}$. Para um determinado número de informações $\mathrm{N}$, os parâmetros $\alpha, \gamma, \beta_{1}, \beta_{2}$ e $\beta_{3}$ são obtidos pelo método dos mínimos quadrados. 
TABELA 1. Parâmetros das relações intensidade-duração-freqüência para alguns municípios do Estado de Goiás. Relationships intensity-duration-frequency for some cities in Goiás.

\begin{tabular}{lcccccc}
\hline Localidade & Latitude & Longitude & $\mathrm{a}$ & $\mathrm{b}$ & \multicolumn{1}{c}{$\mathrm{c}$} & $\mathrm{d}$ \\
\hline Alvorada do Norte & $14^{\circ} 24^{\prime}$ & $46^{\circ} 36^{\prime}$ & 70,7740 & 0,1471 & 29,7 & 0,97194 \\
Alto Paraíso de Goiás & $14^{\circ} 08^{\prime}$ & $47^{\circ} 30^{\prime}$ & 15,8617 & 0,1629 & 12,0 & 0,75990 \\
Aporé & $18^{\circ} 59^{\prime}$, & $52^{\circ} 00^{\prime}$ & 38,4327 & 0,1471 & 18,4 & 0,89575 \\
Aruanã & $14^{\circ} 49^{\prime}$ & $51^{\circ} 10^{\prime}$ & 76,7073 & 0,1471 & 37,4 & 0,96750 \\
Bandeirantes & $13^{\circ} 41^{\prime}$ & $50^{\circ} 48^{\prime}$ & 20,3821 & 0,1702 & 12,0 & 0,75990 \\
Caiapônia & $16^{\circ} 57^{\prime}$, & $51^{\circ} 50^{\prime}$ & 47,9869 & 0,1471 & 20,7 & 0,90521 \\
Campo Alegre de Goiás & $17^{\circ} 40^{\prime}$ & $47^{\circ} 37^{\prime}$ & 22,5868 & 0,1471 & 8,7 & 0,80396 \\
Catalão & $18^{\circ} 11^{\prime}$ & $47^{\circ} 57^{\prime}$ & 29,3749 & 0,1471 & 16,3 & 0,84572 \\
Cavalcante & $13^{\circ} 47^{\prime}$ & $47^{\circ} 27^{\prime}$ & 17,9122 & 0,1040 & 12,0 & 0,75970 \\
Ceres & $15^{\circ} 16^{\prime}$ & $49^{\circ} 34^{\prime}$ & 52,1072 & 0,1471 & 24,6 & 0,92919 \\
Corumbaíba & $18^{\circ} 09^{\prime}$ & $48^{\circ} 34^{\prime}$ & 15,2177 & 0,1525 & 12,0 & 0,75990 \\
Cristalina & $16^{\circ} 45^{\prime}$ & $47^{\circ} 37^{\prime}$ & 14,6369 & 0,2088 & 12,0 & 0,76000 \\
Formosa & $15^{\circ} 32^{\prime}$ & $47^{\circ} 20^{\prime}$ & 24,1153 & 0,1471 & 11,4 & 0,82128 \\
Goiânia & $16^{\circ} 40^{\prime}$ & $49^{\circ} 16^{\prime}$ & 64,3044 & 0,1471 & 24,8 & 0,97471 \\
Israelândia & $16^{\circ} 22^{\prime}$ & $50^{\circ} 54^{\prime}$ & 28,3348 & 0,1471 & 15,3 & 0,82205 \\
Itaberaí & $16^{\circ} 01^{\prime}$ & $49^{\circ} 48^{\prime}$ & 18,1781 & 0,1302 & 12,0 & 0,76000 \\
Itumbiara & $18^{\circ} 45^{\prime}$ & $51^{\circ} 18^{\prime}$ & 15,3126 & 0,1212 & 12,0 & 0,76000 \\
Morrinhos & $17^{\circ} 46^{\prime}$ & $49^{\circ} 08^{\prime}$ & 52,9342 & 0,1471 & 24,9 & 0,91634 \\
Montividiu & $15^{\circ} 58^{\prime}$ & $51^{\circ} 20^{\prime}$ & 16,9765 & 0,1354 & 12,0 & 0,75980 \\
Niquelândia & $14^{\circ} 28^{\prime}$ & $48^{\circ} 27^{\prime}$ & 47,1091 & 0,1471 & 19,5 & 0,90488 \\
Pires do Rio & $17^{\circ} 20^{\prime}$ & $48^{\circ} 15^{\prime}$ & 21,8826 & 0,1471 & 8,0 & 0,79943 \\
Santa Teresinha de Goiás & $14^{\circ} 26^{\prime}$ & $49^{\circ} 42^{\prime}$ & 19,4692 & 0,1715 & 12,0 & 0,76000 \\
\hline
\end{tabular}

Sendo $\mathrm{Z}$ a soma dos quadrados dos desvios e $\mathrm{y}=\mathrm{h}_{(\mathrm{t} ; \mathrm{TR})}$, tem-se que, pela eq.(4):

$$
\mathrm{Z}=\sum \varepsilon^{2}=\sum(\mathrm{y}-\hat{\mathrm{y}})^{2}=\sum\left[\mathrm{y}_{\mathrm{i}}-\mathrm{f}\left(\alpha, \gamma, \beta_{1}, \beta_{2}, \beta_{3}, \mathrm{TR}, \mathrm{t}, \mathrm{h}_{(60 ; 2)}\right)\right]^{2}
$$

em que,

$$
\varepsilon \text { - desvio do ajuste. }
$$

RIGHETTO (1998) apresentou o seguinte procedimento empregado na obtenção das estimativas dos parâmetros, utilizando a expansão de Taylor, bem como inicializações para os parâmetros do modelo de Bell, denotados por $\alpha^{0}, \gamma^{0}, \beta_{1}^{0}, \beta_{2}^{0}, \beta_{3}^{0}$ [eq.(5)]:

$$
\mathrm{f}\left(\alpha, \gamma, \beta_{1}, \beta_{2}, \beta_{3}, \mathrm{TR}, \mathrm{t}, \mathrm{h}_{(60 ; 2)}\right) \cong \mathrm{f}\left(\alpha^{\mathrm{o}}, \gamma^{\mathrm{o}}, \beta_{1}^{\mathrm{o}}, \beta_{2}^{\mathrm{o}}, \beta_{3}^{\mathrm{o}}, \mathrm{TR}, \mathrm{t}, \mathrm{h}_{(60 ; 2)}\right)+\delta_{\mathrm{i}} \frac{\partial \mathrm{f}}{\partial \kappa}
$$

em que,

$\delta-\left(\kappa-\kappa^{0}\right)$

$\kappa$ - parâmetros $\alpha, \gamma, \beta_{1}, \beta_{2}$ e $\beta_{3}$, e

i - 1 a n, parâmetros a serem ajustados.

Fazendo-se $U=\frac{\partial f}{\partial \kappa}$, obtém-se a equação de regressão linear múltipla [(eq. (6)]:

$$
Z_{i}=\left(\alpha-\alpha^{0}\right) U_{1, i}+\left(\beta_{1}-\beta_{1}^{o}\right) U_{2, i}+\left(\beta_{2}-\beta_{2}^{o}\right) U_{3, i}+\left(\gamma-\gamma^{o}\right) U_{4, i}+\left(\beta_{3}-\beta_{3}^{o}\right) U_{5, i}
$$

cujas derivadas parciais são dadas pelas eqs.(7), (8), (9), (10) e (11): 


$$
\begin{aligned}
& \mathrm{U}_{1}=\frac{\partial \mathrm{f}}{\partial \alpha}=\operatorname{Ln}(\mathrm{TR})\left(\beta_{2} \mathrm{t}^{\gamma}-\beta_{3}\right) \mathrm{h}_{(60 ; 2)} \\
& \mathrm{U}_{2}=\frac{\partial \mathrm{f}}{\partial \beta_{1}}=\left(\beta_{2} \mathrm{t}^{\gamma}-\beta_{3}\right) \mathrm{h}_{(60 ; 2)} \\
& \mathrm{U}_{3}=\frac{\partial \mathrm{f}}{\partial \beta_{2}}=\mathrm{t}^{\gamma}\left(\alpha \operatorname{Ln}(\mathrm{TR})+\beta_{1}\right) \mathrm{h}_{(60 ; 2)} \\
& \mathrm{U}_{4}=\frac{\partial \mathrm{f}}{\partial \gamma}=\beta_{2} \mathrm{t}^{\gamma} \operatorname{Ln}(\mathrm{t})\left[\alpha \operatorname{Ln}(\mathrm{TR})+\beta_{1}\right] \mathrm{h}_{(60 ; 2)} \\
& \mathrm{U}_{5}=\frac{\partial \mathrm{f}}{\partial \beta_{3}}=-\left(\alpha \operatorname{Ln}(\mathrm{TR})+\beta_{1}\right) \mathrm{h}_{(60 ; 2)}
\end{aligned}
$$

Aplicando-se o somatório na eq.(6), tem-se um sistema de equações normais [eq. (12)]:

$$
\sum \mathrm{Z}_{\mathrm{i}}=\delta_{1} \sum \mathrm{U}_{1, \mathrm{i}}+\delta_{2} \sum \mathrm{U}_{2, \mathrm{i}}+\delta_{3} \sum \mathrm{U}_{3, \mathrm{i}}+\delta_{4} \sum \mathrm{U}_{4, \mathrm{i}}+\delta_{5} \sum \mathrm{U}_{5, \mathrm{i}}
$$

A partir dos valores iniciais assumidos para os parâmetros do modelo, aplica-se sucessivamente o sistema de equações lineares, de modo a se obterem valores dos desvios cada vez mais próximos de zero. Para cada iteração $\tau$, os valores dos parâmetros assumem os obtidos na iteração anterior, acrescidos dos desvios, ou seja, $\kappa^{\tau+1}=\kappa^{\tau}+\delta$.

Esse desenvolvimento foi implementado computacionalmente, gerando uma rotina de cálculo para o ajuste dos parâmetros do modelo de Bell. Para tal, atribui-se como valores iniciais os obtidos por RIGHETTO (1998) na análise de uma série de estações pluviométricas brasileiras, conforme apresentados na eq.(13).

$$
\mathrm{h}_{(\mathrm{t} ; \mathrm{TR})}=(0,31 \ln \mathrm{TR}+0,70)\left(0,38 \mathrm{t}^{0,31}-0,39\right) \mathrm{h}_{(60 ; 2)}
$$

Como critério de parada para o cálculo iterativo, adotou-se o coeficiente de determinação. A cada iteração, o valor do coeficiente de determinação era comparado com o anterior, e quando se aproximavam da unidade com variações inferiores a $10^{-5}$, o processo iterativo era interrompido. Foram ajustados os parâmetros regionais do modelo de Bell para cada localidade estudada, e um modelo geral para o Estado de Goiás. A partir dos modelos ajustados para cada localidade e geral, calcularam-se as alturas pluviométricas das chuvas para os tempos de duração de $5 ; 10 ; 30 ; 60 ; 90$ e 120 minutos e períodos de retorno de $2 ; 5 ; 10$ e 20 anos. Os valores obtidos foram comparados com os estimados pelas relações intensidade-duração-freqüência geradas por COSTA et al. (2001) e OLIVEIRA et al. (2005) e pelo modelo ajustado por RIGHETTO (1998), empregando-se, para tal, o erro-padrão da estimativa.

Para as localidades do Estado de Goiás desprovidas de registros pluviométricos, o modelo de Bell pode ser ajustado a partir da regionalização dos parâmetros obtidos. Para isso, empregou-se, na regionalização dos coeficientes do modelo de Bell, os softwares Surfer, versão 6.04, e o Idrisi versão 32. Utilizou-se, na regionalização, um retângulo envolvente com latitude variando entre $12 \mathrm{e}$ $20^{\circ} \mathrm{S}$ e longitude entre $45^{\circ} 30^{\prime}$ e $53^{\circ} 30^{\prime}$ ' W, dividido em 2.000 colunas e 2.000 linhas, com pixel de 14,4". O interpolador empregado na regionalização foi a krigagem, devido as suas características desejáveis como estimador, ou seja, ser não-tendencioso e apresentar variância mínima (CARVALHO \& VIEIRA, 2001). Na interpolação por krigagem, foi avaliado o desempenho dos modelos de semivariogramas esférico, gaussiano e exponencial, empregando, para tal, o coeficiente de Willmott [eq.(14)], descrito por CORTÊS (2004). 


$$
\mathrm{d}=100\left[1-\frac{\sum\left(\mathrm{o}_{\mathrm{i}}-\mathrm{e}_{\mathrm{i}}\right)^{2}}{\sum\left(\left|\hat{\mathrm{o}}_{\mathrm{i}}\right|+\left|\hat{\mathrm{e}}_{\mathrm{i}}\right|\right)^{2}}\right]
$$

em que,

d - coeficiente de desempenho de Willmott, \%;

$\mathrm{o}_{\mathrm{i}}$ e $\mathrm{e}_{\mathrm{i}}$ - valores observados e estimados, respectivamente;

$\hat{o}_{i}-o_{i}-o_{m}$ e $\hat{\mathrm{e}}_{i}=e_{i}-e_{m}, \mathrm{e}$

$\mathrm{o}_{\mathrm{m}}$ e $\mathrm{e}_{\mathrm{m}}$ - média dos valores observados e estimados, respectivamente.

$\mathrm{Na}$ análise do desempenho dos modelos de semivariograma, empregou-se a classificação apresentada por CAMARGO \& SENTELHAS (1997), apresentada na Tabela 2.

TABELA 2. Classificação do coeficiente de desempenho de Willmott. Willmott's performance coefficient classification.

\begin{tabular}{cc}
\hline Coeficiente de Desempenho de Willmott (\%) & Classificação \\
\hline$>85$ & Ótimo \\
76 a 85 & Muito bom \\
66 a 75 & Bom \\
61 a 65 & Mediano \\
51 a 60 & Sofrível \\
41 a 50 & Mau \\
$\leq 40$ & Péssimo \\
\hline
\end{tabular}

Empregando o banco de dados da Agência Nacional das Águas (ANA, 2006) de chuvas diárias, observadas no período de 1979 a 1994, foram construídas, para todas as localidades estudadas, as séries anuais dos valores máximos de chuva. Para cada série de valores extremos, verificou-se a aderência da distribuição de Gumbel [eq.(15)], pelo teste de Kolmogorov-Sminorv, para o nível de significância de 5\%.

$$
\mathrm{h}_{\mathrm{TR}}=\mathrm{a}-\mathrm{bLn}\left[\operatorname{Ln}\left(\frac{\mathrm{TR}}{\mathrm{TR}-1}\right)\right]
$$

em que,

$\mathrm{h}_{\mathrm{TR}}$ - altura pluviométrica de um dia associado a um período de retorno TR, mm, e

a e b - parâmetros da distribuição de Gumbel, obtidos pelo método dos momentos [eq. (16)]:

$$
\mathrm{E}(\mathrm{h})=\mathrm{a}+0,577 \mathrm{~b} \text { e } \operatorname{VAR}(\mathrm{h})=1,645 \mathrm{~b}^{2}
$$

em que,

$\mathrm{E}(\mathrm{h})$ - valor esperado estimado pela média dos valores máximos anuais, e

VAR(h) - variância dos valores máximos anuais, estimada a partir da série de dados.

Empregando-se a distribuição de Gumbel, calculou-se, para cada localidade, a altura pluviométrica de um dia de duração, para o período de retorno de dois anos. As alturas das chuvas máximas $\mathrm{h}_{(60,2)}$ e $\mathrm{h}_{(\mathrm{dia}, 2)}$ foram regionalizadas, permitindo a obtenção do mapa temático dos coeficientes $\mathrm{K}$, pelo cruzamento dos mapas $\mathrm{h}_{(60,2)} \mathrm{e}_{(\mathrm{dia}, 2)}$, empregando-se, no software Idrisi, a ferramenta Overlay, a qual permite a operação de divisão dos referidos mapas. O mapa temático gerado pelo cruzamento dos mapas de $\mathrm{h}_{(60,2)}$ e $\mathrm{h}_{(\mathrm{dia}, 2)}$ permite a obtenção do valor de $\mathrm{K}$ para as localidades desprovidas de registros pluviométricos.

\section{RESULTADOS E DISCUSSÃO}

$\mathrm{Na}$ Tabela 3, apresentam-se os coeficientes ajustados do modelo de Bell para as diferentes localidades estudadas e para o modelo geral do Estado de Goiás. Observa-se, na referida Tabela, 
adequado ajustamento dos modelos, com coeficientes de determinação próximos de 1,0, permitindo a utilização dos modelos nas localidades para as quais os mesmos foram ajustados, com pequena margem de erro na estimativa das alturas máximas precipitadas, para durações inferiores a 120 minutos, e com período de retorno menores que 20 anos. Os coeficientes apresentados na Tabela 3 foram diferentes dos obtidos por RIGHETTO (1998), na análise de uma série de estações pluviométricas brasileiras, ressaltando a importância de estudos regionais no ajustamento dos coeficientes do modelo de Bell.

TABELA 3. Coeficientes ajustados do modelo de Bell para diferentes localidades, e geral, para o Estado de Goiás. Adjusted coefficients of Bell model for different locations in Goiás and for the state in general.

\begin{tabular}{|c|c|c|c|c|c|c|}
\hline Município & $\alpha$ & $\beta_{1}$ & $\beta_{2}$ & $\beta_{3}$ & $\gamma$ & $r^{2}$ \\
\hline Alto Paraíso de Goiás & 0,1555 & 0,5984 & 2,8563 & 3,0364 & 0,1022 & $0,9928^{*}$ \\
\hline Alvorada do Norte & 0,1320 & 0,6512 & 1,7504 & 1,9803 & 0,1546 & $0,9996^{*}$ \\
\hline Aporé & 0,1082 & 0,5377 & 4,0324 & 4,3000 & 0,0931 & $0,9997^{*}$ \\
\hline Aruanã & 0,0603 & 0,2975 & 1,9799 & 2,4011 & 0,2396 & $0,9996^{*}$ \\
\hline Bandeirantes & 0,1112 & 0,4068 & 2,1706 & 2,3647 & 0,1667 & $0,9960^{*}$ \\
\hline Caiapônia & 0,0984 & 0,4911 & 3,5074 & 3,8038 & 0,1125 & $0,9997^{*}$ \\
\hline Campo Alegre & 0,1394 & 0,7011 & 3,8207 & 3,9283 & 0,0739 & $0,9999^{*}$ \\
\hline Catalão & 0,1047 & 0,5225 & 2,7162 & 2,9449 & 0,1287 & $0,9998^{*}$ \\
\hline Cavalcante & 0,0691 & 0,5098 & 1,7271 & 1,8839 & 0,1800 & $0,9985^{*}$ \\
\hline Ceres & 0,0944 & 0,4713 & 2,8789 & 3,1923 & 0,1358 & $0,9997^{*}$ \\
\hline Corumbaíba & 0,0896 & 0,4286 & 1,9093 & 2,0830 & 0,1865 & $0,9999^{*}$ \\
\hline Cristalina & 0,1287 & 0,3918 & 2,0456 & 2,2312 & 0,1799 & $0,9998^{*}$ \\
\hline Formosa & 0,1076 & 0,5368 & 3,9944 & 4,1795 & 0,0909 & $0,9999^{*}$ \\
\hline Goiânia & 0,1319 & 0,6585 & 3,8593 & 4,1221 & 0,0833 & $0,9997^{*}$ \\
\hline Israelândia & 0,1088 & 0,5432 & 2,1684 & 2,3658 & 0,1471 & $0,9999^{*}$ \\
\hline Itaberaí & 0,0598 & 0,3521 & 2,3506 & 2,5645 & 0,1882 & $0,9999^{*}$ \\
\hline Itumbiara & 0,0567 & 0,3655 & 2,2748 & 2,4818 & 0,1889 & $0,9999^{*}$ \\
\hline Morrinhos & 0,1020 & 0,5091 & 2,2242 & 2,4996 & 0,1548 & $0,9997^{*}$ \\
\hline Montividiu & 0,0532 & 0,2982 & 2,7629 & 3,0146 & 0,1881 & $0,9999^{*}$ \\
\hline Niquelândia & 0,1184 & 0,5908 & 3,5760 & 3,8272 & 0,0955 & $0,9998^{*}$ \\
\hline Pires do Rio & 0,1343 & 0,6703 & 3,8775 & 3,9717 & 0,0749 & $0,9999^{*}$ \\
\hline Santa Teresinha de Goiás & 0,0951 & 0,3873 & 2,0983 & 2,2891 & 0,1845 & $0,9999^{*}$ \\
\hline$\overline{\text { Geral }}$ & 0,1087 & 0,5386 & 2,2125 & 2,4075 & 0,1448 & $0,9971^{*}$ \\
\hline
\end{tabular}

* significativo para $5 \%$ de probabilidade, pela distribuição de $\mathrm{F}$.

Analisando as estimativas das alturas máximas precipitadas para os tempos de duração de 5; 10; 30; 60; 90 e 120 minutos, e períodos de retorno de 2 ; 5; 10 e 20 anos, verificou-se que os errospadrão das estimativas foram, em média, de 4,84; 4,97 e 8,59 quando se empregaram os modelos locais, geral para o Estado de Goiás e ajustado por RIGHETTO (1998), respectivamente (Tabela 4). As estimativas empregando-se os modelos locais e geral não apresentaram grandes diferenças, permitindo, assim, a utilização do modelo geral para as localidades do Estado de Goiás desprovidas de séries históricas de longa duração, exigindo, para isso, pelo menos dois anos de observações, para a determinação da altura pluviométrica da chuva com 60 minutos de duração.

Para as localidades do Estado de Goiás desprovidas de registros pluviométricos, o modelo de Bell pode ser ajustado pela obtenção dos coeficientes regionalizados, extraídos dos mapas temáticos apresentados na Figura 1. Não se verificaram diferenças nas interpolações dos coeficientes regionalizados quando se empregaram os semivariogramas gaussiano, esférico e exponencial, com coeficientes de Willmott próximos de 100,0\%. 
TABELA 4. Erro-padrão das alturas precipitadas estimadas pelos modelos de Bell, ajustado para as diferentes localidades, geral e por RIGHETTO (1998). Standard error from the rainfall highs estimated with Bell models, adjusted for different locations, for the state in general and by RIGHETTO (1998).

\begin{tabular}{lccc}
\hline Município & Estação & Geral & RIGHETTO (1998) \\
\hline Alto Paraíso de Goiás & 3,24 & 3,46 & 4,83 \\
Alvorada do Norte & 6,84 & 7,46 & 11,73 \\
Aporé & 5,91 & 5,78 & 9,76 \\
Aruanã & 6,95 & 8,29 & 12,26 \\
Bandeirantes & 3,15 & 3,66 & 4,83 \\
Caiapônia & 6,86 & 6,91 & 11,46 \\
Campo Alegre & 5,80 & 5,30 & 9,26 \\
Catalão & 5,74 & 5,53 & 9,36 \\
Cavalcante & 1,46 & 3,63 & 7,57 \\
Ceres & 6,40 & 6,73 & 10,87 \\
Corumbaíba & 5,83 & 5,14 & 8,46 \\
Cristalina & 8,84 & 4,70 & 7,14 \\
Formosa & 5,57 & 5,09 & 8,90 \\
Goiânia & 6,45 & 6,71 & 10,99 \\
Israelândia & 6,20 & 5,93 & 10,04 \\
Itaberaí & 0,39 & 1,06 & 5,57 \\
Itumbiara & 0,31 & 1,37 & 5,15 \\
Morrinhos & 6,86 & 7,29 & 11,66 \\
Montividiu & 0,38 & 0,73 & 4,92 \\
Niquelândia & 6,85 & 6,80 & 11,39 \\
Pires do Rio & 5,82 & 5,32 & 9,24 \\
Santa Teresinha de Goiás & 0,59 & 2,52 & 3,48 \\
\hline Média & 4,84 & 4,97 & 8,59 \\
\hline
\end{tabular}

(a) coeficiente $\alpha$

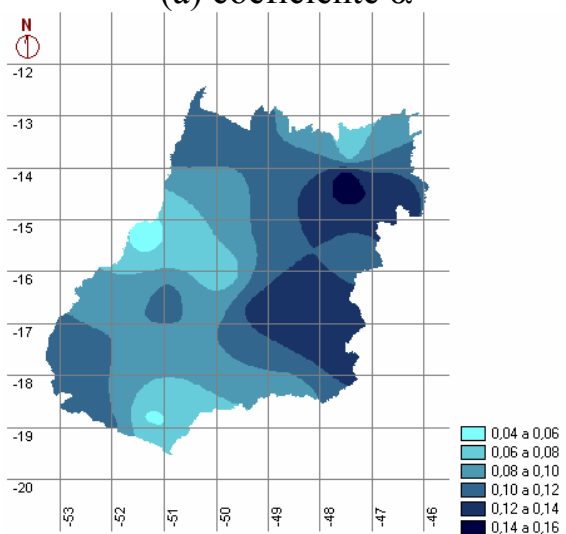

(d) coeficiente $\beta_{3}$

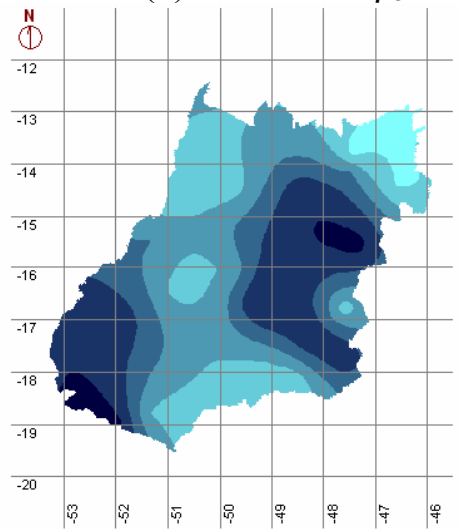

(b) coeficiente $\beta_{1}$

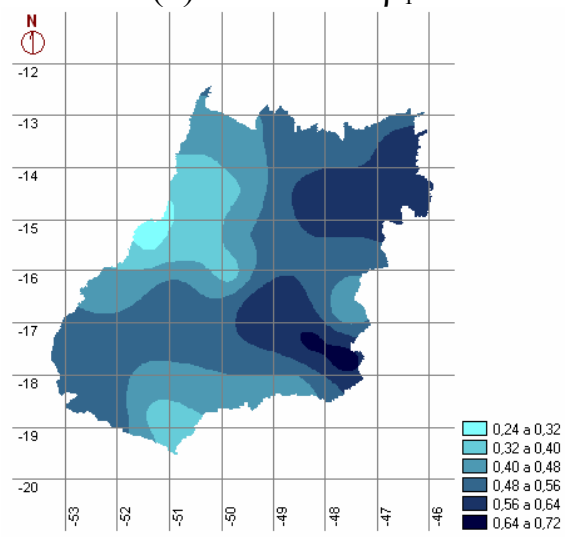

(c) coeficiente $\beta_{2}$

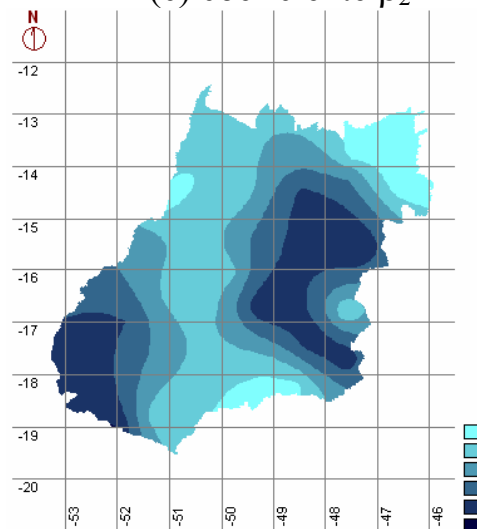

(e) coeficiente $\gamma$

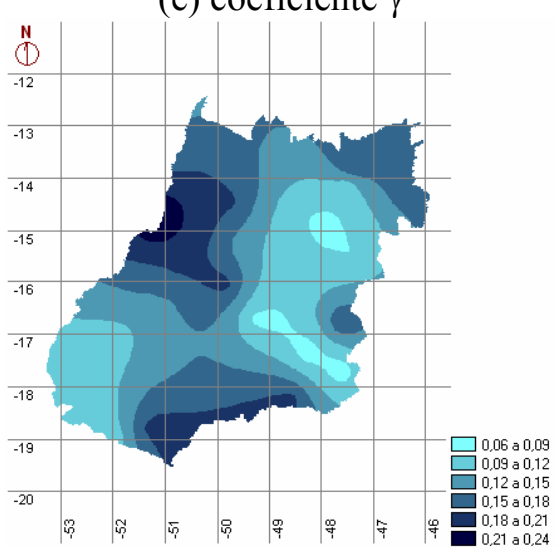

FIGURA 1. Mapas temáticos dos coeficientes do modelo de Bell. Thematic maps from the Bell model coefficients. 
Na Figura 2, apresentam-se os mapas temáticos das alturas de chuvas $\mathrm{h}_{(60,2)}$ e $\mathrm{h}_{(\mathrm{dia}, 2)}$ e do coeficiente K para o Estado de Goiás, o que permite a sua obtenção para as localidades desprovidas de registros pluviográficos, uma vez conhecidas as suas coordenadas geográficas. Na regionalização dos coeficientes $\mathrm{K}$, também não se verificaram grandes diferenças quando se empregaram os semivariogramas gaussiano, esférico e exponencial (Tabela 5), com coeficientes de Willmott próximos de $100 \%$. Os parâmetros dos semivariogramas para os modelos gaussiano, esférico e exponencial estão apresentados na Tabela 5, na qual se podem observar pequenas diferenças nos parâmetros ajustados.

(a) altura de chuva $\mathrm{h}_{(60,2)}$

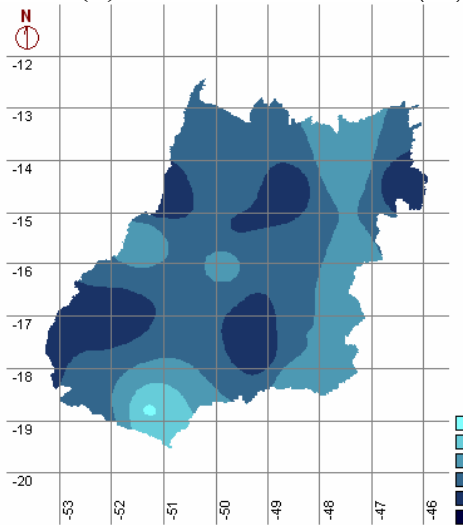

(b) altura de chuva $\mathrm{h}_{(\mathrm{dia}, 2)}$

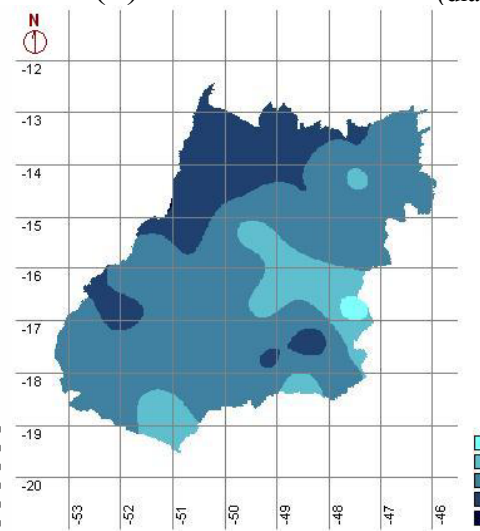

(c) coeficiente $\mathrm{K}$

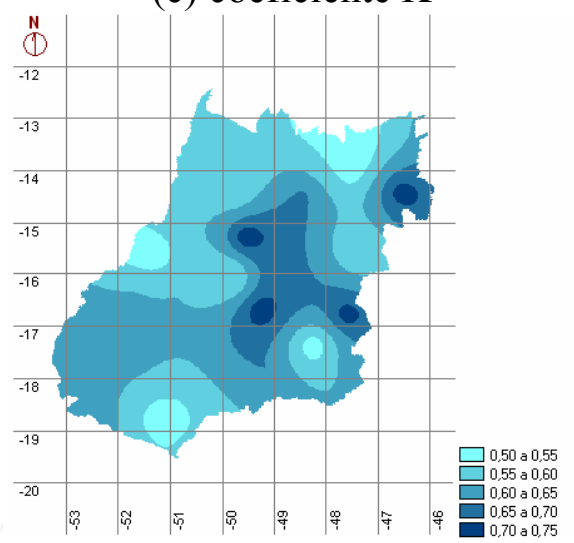

FIGURA 2. Mapas temáticos das alturas de chuva $\mathrm{h}_{(60,2)}$ e $\mathrm{h}_{(\mathrm{dia}, 2)}$ e do coeficiente $\mathrm{K}$. Thematic maps from the $h_{(60,2)}$ and $h_{(d i a, 2)}$ rainfall and $K$ coefficient.

TABELA 5. Coeficientes $\mathrm{K}_{\mathrm{c}}$ calculados pela relação entre $\mathrm{h}_{(60,2)}$ e $\mathrm{h}_{(\mathrm{dia}, 2)}$ e $\mathrm{K}_{\mathrm{k}}$ obtidos por krigagem, para os diferentes municípios de Goiás, e parâmetros do semivariograma. Coefficients $\mathbf{K}_{\mathbf{c}}$ calculated by the relation between $h\left(_{60,2}\right)$ and $h\left({ }_{d a y, 2}\right)$ and $K_{k}$ estimated from kriging, for the different cities in Goiás, and the semivariogram parameters.

\begin{tabular}{lrrrrrr}
\hline \multirow{2}{*}{ Município } & $\mathrm{h}_{(60,2)}$ & $\mathrm{h}_{(\mathrm{dia}, 2)}$ & \multirow{2}{*}{$\mathrm{K}_{\mathrm{c}}$} & \multicolumn{3}{c}{$\mathrm{K}_{\mathrm{k}}$} \\
\cline { 6 - 7 }$(\mathrm{mm})$ & $(\mathrm{mm})$ & & Gaussiano & Esférico & Exponencial \\
\hline Alto Paraíso de Goiás & 41,32 & 77,31 & 0,5345 & 0,5343 & 0,5345 & 0,5345 \\
Alvorada do Norte & 59,47 & 81,65 & 0,7284 & 0,7282 & 0,7283 & 0,7283 \\
Aporé & 51,32 & 85,22 & 0,6022 & 0,6007 & 0,6021 & 0,6022 \\
Aruanã & 60,73 & 102,71 & 0,5913 & 0,5889 & 0,5907 & 0,5904 \\
Bandeirantes & 53,36 & 97,14 & 0,5493 & 0,5447 & 0,5493 & 0,5493 \\
Caiapônia & 59,90 & 91,04 & 0,6580 & 0,6606 & 0,6577 & 0,6577 \\
Campo Alegre de Goiás & 50,05 & 80,20 & 0,6241 & 0,6195 & 0,6233 & 0,6234 \\
Catalão & 49,93 & 83,25 & 0,5998 & 0,5992 & 0,5997 & 0,5998 \\
Cavalcante & 44,71 & 89,25 & 0,5010 & 0,5034 & 0,5017 & 0,5017 \\
Ceres & 56,03 & 75,19 & 0,7452 & 0,7427 & 0,7437 & 0,7437 \\
Corumbaíba & 45,26 & 72,75 & 0,6221 & 0,6229 & 0,6221 & 0,6221 \\
Cristalina & 47,64 & 64,72 & 0,7361 & 0,7358 & 0,7350 & 0,7349 \\
Formosa & 48,12 & 90,09 & 0,5341 & 0,5310 & 0,5346 & 0,5346 \\
Goiânia & 56,37 & 75,21 & 0,7495 & 0,7468 & 0,7480 & 0,7484 \\
Israelândia & 53,94 & 90,02 & 0,5992 & 0,5997 & 0,5993 & 0,5993 \\
Itaberaín & 46,27 & 89,66 & 0,5161 & 0,5160 & 0,5171 & 0,5170 \\
Itumbiara & 38,74 & 74,52 & 0,5199 & 0,5186 & 0,5203 & 0,5202 \\
Morrinhos & 60,07 & 92,43 & 0,6499 & 0,6505 & 0,6494 & 0,6495 \\
Montividiu & 43,41 & 86,04 & 0,5045 & 0,5052 & 0,5052 & 0,5052 \\
Niquelândia & 59,69 & 90,32 & 0,6609 & 0,6649 & 0,6608 & 0,6608 \\
Pires do Rio & 49,84 & 97,17 & 0,5129 & 0,5146 & 0,5143 & 0,5142 \\
Santa Teresinha de Goiás & 51,00 & 92,69 & 0,5502 & 0,5500 & 0,5503 & 0,5503 \\
\hline Média & & & 0,6040 & 0,6036 & 0,6040 & 0,6040 \\
\hline Efeito pepita & & & & 0 & 0 & 0 \\
Patamar & & & & 0,00977 & 0,00961 & 0,01296 \\
Alcance (m) & & & & & & 93737,38 \\
\hline
\end{tabular}


Para verificar a qualidade da regionalização, selecionaram-se 20 municípios do Estado de Goiás, para os quais foram extraídos da Figura 1 os coeficientes para a reconstrução do modelo de Bell. Os valores dos coeficientes do modelo de Bell para os referidos municípios estão apresentados na Tabela 6, bem como o erro-padrão da estimativa (EP) obtidos em relação às alturas das chuvas máximas diárias, determinadas a partir da Figura 2b. Para todos os municípios investigados, as alturas de chuvas estimadas pelo modelo ajustado, com base na regionalização, apresentaram erropadrão da estimativa médio de 3,57 , com valores variando entre 0,70 para o municio de Goiás e 6,95 para Caldas Novas. Os valores dos erros-padrão de estimativa encontrados para os 20 municípios investigados estão dentro da faixa encontrada para os municípios com registros pluviográficos, e inferiores aos obtidos pelo modelo geral ajustado por RIGHETTO (1998), apresentados na Tabela 4.

TABELA 6. Coeficientes do modelo de Bell obtidos pela regionalização e erros-padrão da estimativa. Bell model coefficients obtained from regionalization and standard error of estimate.

\begin{tabular}{lcccccccc}
\hline Município & Latitude & Longitude & $\alpha$ & $\beta_{1}$ & $\beta_{2}$ & $\beta_{3}$ & $\gamma$ & EP \\
\hline Anápolis & $16^{\circ} 05^{\prime}$ & $48^{\circ} 30^{\prime}$ & 0,1247 & 0,5551 & 3,6104 & 3,8321 & 0,1017 & 4,86 \\
Balisa & $16^{\circ} 15^{\prime}$ & $52^{\circ} 30^{\prime}$ & 0,0891 & 0,4396 & 3,2811 & 3,5525 & 0,1351 & 4,94 \\
Caldas Novas & $17^{\circ} 43^{\prime}$ & $48^{\circ} 31^{\prime}$ & 0,1100 & 0,5560 & 2,8307 & 2,9972 & 0,1304 & 6,95 \\
Campos Belos & $13^{\circ} 01^{\prime}$ & $46^{\circ} 46^{\prime}$ & 0,0823 & 0,5416 & 1,7407 & 1,9240 & 0,1790 & 1,28 \\
Edéia & $17^{\circ} 18^{\prime}$ & $49^{\circ} 55^{\prime}$, & 0,1005 & 0,5708 & 2,5021 & 2,7454 & 0,1447 & 6,60 \\
Flores de Goiás & $14^{\circ} 27^{\prime}$ & $47^{\circ} 02^{\prime}$ & 0,1369 & 0,6128 & 2,4245 & 2,6387 & 0,1277 & 3,17 \\
Goianésia & $15^{\circ} 19^{\prime}$ & $49^{\circ} 07^{\prime}$ & 0,1036 & 0,5111 & 3,2334 & 3,5073 & 0,1203 & 2,94 \\
Goiás & $15^{\circ} 56^{\prime}$ & $50^{\circ} 08^{\prime}$ & 0,0696 & 0,3873 & 2,2608 & 2,4935 & 0,1836 & 0,70 \\
Itajá & $19^{\circ} 07^{\prime}$ & $51^{\circ} 38^{\prime}$ & 0,0845 & 0,4081 & 3,2173 & 3,4593 & 0,1384 & 2,96 \\
Jataí & $17^{\circ} 55^{\prime}$ & $51^{\circ} 45^{\prime}$ & 0,0939 & 0,4845 & 3,2314 & 3,4717 & 0,1247 & 3,29 \\
Monte Alegre de Goiás & $13^{\circ} 05^{\prime}$ & $46^{\circ} 53^{\prime}$ & 0,0810 & 0,5441 & 1,7526 & 1,9318 & 0,1784 & 1,28 \\
Planaltina & $15^{\circ} 27^{\prime}$ & $47^{\circ} 36^{\prime}$ & 0,1219 & 0,5635 & 3,8962 & 4,0935 & 0,0910 & 2,73 \\
Pontalina & $17^{\circ} 30^{\prime}$ & $49^{\circ} 26^{\prime}$ & 0,1056 & 0,5803 & 2,5321 & 2,7900 & 0,1407 & 5,93 \\
Posse & $14^{\circ} 06^{\prime}$ & $46^{\circ} 22^{\prime}$ & 0,1154 & 0,6220 & 1,7350 & 1,9538 & 0,1617 & 1,28 \\
Rio Verde & $17^{\circ} 48^{\prime}$ & $50^{\circ} 55^{\prime}$ & 0,0851 & 0,4719 & 2,5916 & 2,8166 & 0,1502 & 2,58 \\
Quirinópolis & $18^{\circ} 34^{\prime}$ & $50^{\circ} 34^{\prime}$ & 0,0678 & 0,4313 & 2,1419 & 2,3398 & 0,1842 & 2,25 \\
São Domingos & $13^{\circ} 24^{\prime}$ & $46^{\circ} 19^{\prime}$ & 0,0974 & 0,5643 & 1,7813 & 1,9788 & 0,1695 & 0,93 \\
São Félix & $13^{\circ} 31^{\prime}$ & $48^{\circ} 09^{\prime}$ & 0,0947 & 0,5293 & 2,4389 & 2,6097 & 0,1438 & 3,32 \\
São Miguel do Araguaia & $13^{\circ} 16^{\prime}$ & $50^{\circ} 10^{\prime}$ & 0,1099 & 0,4342 & 2,2240 & 2,4025 & 0,1618 & 2,17 \\
Três Ranchos & $18^{\circ} 25^{\prime}$ & $47^{\circ} 48^{\prime}$ & 0,1067 & 0,5825 & 2,6878 & 2,9096 & 0,1307 & 5,24 \\
\hline
\end{tabular}

\section{CONCLUSÕES}

Para os municípios do Estado de Goiás, com disponibilidade das relações intensidadeduração-freqüência, o modelo de Bell ajustou-se adequadamente, apresentando-se, portanto, como alternativa na obtenção das alturas de chuvas intensas quando se dispõe de séries históricas com poucos anos de observação, sendo mais adequada do que o modelo geral ajustado para todo o território brasileiro. A reconstrução do modelo de Bell para qualquer localidade inserida no Estado de Goiás, obtida a partir dos mapas temáticos dos coeficientes ajustados, permitiu a ampliação das equações que expressam a relação entre a altura de chuva máxima para diferentes durações e período de retorno, com baixos valores no erro-padrão da estimativa.

\section{REFERÊNCIAS}

ANA. AGÊNCIA NACIONAL DAS ÁGUAS. Hidro Web: sistemas de informações hidrológicas. Disponível em: http://hidroweb.ana.gov.br. Acesso em: 10 mar. 2006.

BARBOSA, F.O.A.; OLIVEIRA, L.F.C.; CORTÊS, F.C.; ROMÃO, P.A.; CARVALHO, D.F. Obtenção de equações de chuva intensa para algumas localidades no Estado de Goiás: método da 
desagregação de chuvas. In: CONGRESSO BRASILEIRO DE ENGENHARIA AGRÍCOLA, 29., 2000, Fortaleza. Anais... Fortaleza: Sociedade Brasileira de Engenharia Agrícola, 2000. 1 CDROM.

BELL, F.G. Generalized rainfall-duration- frequency relationships. Journal of the Hydraulics Division, Reston, v.95, n.1, p.311-27, 1969.

BERTONI, J.C.; TUCCI, C.E.M. Precipitação. In: TUCCI, C.E.M. Hidrologia ciência e aplicação. Porto Alegre: ABRH, 1993. p.177-242.

CAMARGO, A.P.; SENTELHAS, P.C. Avaliação do desempenho de diferentes métodos de estimativa da evapotranspiração potencial no Estado de São Paulo, Brasil. Revista Brasileira de Agrometeorologia, Santa Maria, v.5, n.1, p.89-97, 1997.

CARVALHO, J.R.P.; VIEIRA, S.R. Avaliação e comparação de estimadores de krigagem para variáveis agronômicas. Campinas: Embrapa Informática Agropecuária, 2001. 24 p.

CORTÊS, F.C. Obtenção e validação de modelos de regionalização de vazão na bacia do rio dos Bois, Estado de Goiás. 2004. 56 f. Dissertação (Mestrado em Ciências Agrárias) - Universidade de Brasília, Brasília, 2004.

COSTA, A.R.; SANTOS, A.C.C; OLIVEIRA, L.F.C. Regionalização de equações de chuva no cerrado. In: SIMPÓSIO BRASILEIRO DE RECURSOS HÍDRICOS, 5., 2001, Aracaju. Anais... Aracaju: Associação Brasileira de Recursos Hídricos, 2001.1 CD-ROM.

COSTA, A.R.; BRITO, V.F. Equações de chuva intensa para Goiás e sul de Tocantins. In: SIMPÓSIO BRASILEIRO DE RECURSOS HÍDRICOS, 13., 1999. Belo Horizonte. Anais... Associação Brasileira de Recursos Hídricos, 1999. 1 CD-ROM.

COSTA, A.R.; RODRIGUES, A.A. Método das isozonas: desvios entre resultados. In: SIMPÓSIO BRASILEIRO DE RECURSOS HÍDRICOS, 13., 1999, Belo Horizonte. Anais... Belo Horizonte, ABRH, 1999. 1 CD-ROM.

FENDRICH, R. Chuvas intensas para obras de drenagem no Estado do Paraná. Curitiba: Champagnat, 1998. 99 p.

GENOVEZ, A.M.; ZUFFO, A.C. Chuvas intensas no Estado de São Paulo: estudos existentes e análise comparativa. Revista Brasileira de Recursos Hidricos, Porto Alegre, v.5, n.3, p.45-58, 2000.

MARTINEZ JÚNIOR, F. Análise das precipitações intensas no Estado de São Paulo. In: SIMPÓSIO BRASILEIRO DE RECURSOS HÍDRICOS, 13., 1999, Belo Horizonte. Anais... Associação Brasileira de Recursos Hídricos, 1999. 1 CD-ROM.

OLIVEIRA, L.F.C.; CORTÊS, F.C.; WEHR, T.R.; BORGES, L.B.; SARMENTO, P.H.P.; GRIEBELER, N.P. Intensidade-duração-freqüência de chuvas intensas para algumas localidades no Estado de Goiás e Distrito Federal. Pesquisa Agropecuária Tropical, Goiânia, v.35, n.1, p.13-18, 2005.

PFAFSTETTER, O. Chuvas intensas no Brasil. Brasília: Departamento Nacional de Obras e Saneamento, 1957. $246 \mathrm{p}$.

PINTO, F.A.; FERREIRA, P.A.; PRUSKI, F.F.; ALVES, A.R.; CECON, P.R. Equações de chuvas intensas para algumas localidades do Estado de Minas Gerais. Engenharia na Agricultura, Viçosa, v.16, n.1, p.91-104. 1999.

RIGHETTO, A.M. Hidrologia e recursos hídricos. São Carlos: EESC/USP, 1998. 840 p. SILVA, D.D.; VALVERDE, A.E.L.; PRUSKI, F.F.; GONÇALVES, R.A.B. Estimativa e espacialização dos parâmetros da equação de intensidade-duração-freqüência da precipitação para o Estado de São Paulo. Engenharia na Agricultura, Viçosa, v.7, n.2, p.70-87, 1999a. 
SILVA, D.D.; PINTO, F.R.L.; PRUSKI, F.F.; PINTO, F.A. Estimativa e espacialização dos parâmetros da equação de intensidade-duração-freqüência da precipitação para o Rio de Janeiro e o Espírito Santo. Engenharia Agrícola, Jaboticabal, v.18, n.3, p.22-33, 1999 b.

SILVA, D.D.; GOMES FILHO, R.R.; PRUSKI, F.F.; PEREIRA, S.B.; NOVAES, L.F. Chuvas intensas no Estado da Bahia. Revista Brasileira de Engenharia Agrícola e Ambiental, Campina Grande, v.6, n.2, p.362-7, 2002.

SILVA, D.D.; PEREIRA, S.B.; PRUSKI, F.F.; GOMES FILHO, R.R.; LANA, A.M.Q.; BAENA, L.G.N. Equações de intensidade-duração-freqüência da precipitação pluvial para o Estado de Tocantins. Engenharia na Agricultura, Viçosa, v.11, n.1-4, p.7-14, 2003. 